\title{
Grad's 13 moments approximation for Enskog-Vlasov equation
}

\author{
Henning Struchtrup ${ }^{1, a)}$ and Aldo Frezzotti ${ }^{2, b)}$ \\ ${ }^{1}$ Dept. of Mechanical Engineering, University of Victoria, PO Box 1700, Stn. CSC, Victoria, BC, V8W 2Y2, Canada \\ ${ }^{2}$ Dipartimento di Scienze e Tecnologie Aerospaziali, Politecnico di Milano, Via La Masa 34, 20156 Milano, Italy \\ a)struchtr@uvic.ca \\ b)aldo.frezzotti@polimi.it
}

\begin{abstract}
Hydrodynamic models of liquid-vapor flows have to face the difficulty of describing non-equilibrium regions next to interfaces. Depending on the flow regimes and the underlying theoretical models, different answers have been given. In particular, diffuse interface models (DIMs) provide, in principle, a unified description of the whole flow field by a set of PDE's, not much more complex than Navier-Stokes-Fourier classical equations. Unfortunately, DIMs fail to provide a proper description of kinetic layers next to interfaces. In order to develop a model incorporating kinetic effects while keeping the relative simplicity of DIMs, macroscopic transport equations - moment equations — are derived from the Enskog-Vlasov equation. The Enskog-Vlasov equation extends the Enskog equation by accounting for the attractive forces between the gas molecules. Hence, it gives a vander-Waals-like kinetic description of a non-ideal gas, including liquid-vapor phase change. Specifically, the equation describes the liquid phase, the vapor phase, and a diffusive transition region connecting both phases. While not the most accurate model, solutions of the Enskog-Vlasov equation exhibits all relevant phenomena occurring in the evaporation and condensation of rarefied or dense vapors. In this work, Grad's moment method is used to derive a closed set of 13 moment equations. In the appropriate limits, these reduce to the Navier-Stokes-Fourier system for liquid and vapor. Our main interest is to study non-hydrodynamic effects, in particular transport in and across the transition region, and the interplay between the transition region and Knudsen layers. We present first results of this program, including the closed transport equations for 13 moments, discussion of the limits, and solutions in simple geometries.
\end{abstract}

\section{INTRODUCTION}

The Boltzmann equation is valid only for sufficiently diluted gases, where all interactions between particles are binary collisions. Attractive forces are considered during the short collision only, and the finite size of the particles is ignored in the collision details. However, in a dense gas, and in the liquid phase, the average particle distance is so small that the attractive forces of other particles must not be ignored at all times. Moreover, due to finite size and short distances, the interactions are non-binary. Nevertheless, in the Enskog-Vlasov (EV) model one still considers binary collisions only, and describes the attractive interaction with the sea of particles as an external force by summing up the contributions based on particle distance in the Vlasov term [1]. The finite size of particles is considered in the binary collision term due to Enskog [2], which describes collisions of hard sphere particles. With this, both attractive and repulsive forces are in the model, so that dense gas effects and phase change can be described. Despite the simplifications, in particular consideration of only binary collisions, the model works very well, and hence opens a pathway to the study of evaporation and condensation processes.

The model yields van-der-Waals-like equations of state for pressure and energy with full phase change, and diffusive phase interfaces with surface tension $[3,4,5,6]$. Solutions can be obtained by DSMC and other methods for numerically solving the Boltzmann equation. An alternative approach, as followed here, is the construction of macroscopic approximations, i.e., moment equations at higher order, and their solution.

The most fundamental macroscopic approximation, the Navier-Stokes-Fourier equations (NSF) of classical hydrodynamics, were derived before from the Chapman-Enskog expansion [7] of the Enskog equation. Higher order moment equations go beyond the classical hydrodynamics, and describe interesting rarefaction effects such as velocity slip and temperature jumps, thermal stresses, flows induced by temperature gradients (transpiration flow) and 
Knudsen layers [8, 9]. Grad-style [10] 13 moment equations (G13) for the Enskog equation (that is without the Vlasov term) were derived by Kremer and Rosa, who considered only the leading terms in linearized equations [11]. As will be seen below, the addition of the Vlasov term is straightforward, but, in order to properly describe phase change and interfaces, more terms must be considered in expansions, and linearization must rely on non-homogeneous ground states.

The phase field description of the interface by the EV equation, and the NSF and G13 equations derived from it, resolves the phase interface, which has a thickness of a few particle diameters. In non-equilibrium processes we expect a Knudsen layer in the vapor adjacent to the interface with a thickness of the order of the mean free path [8]. Hence, if the interface itself is resolved, one must aim for models that also resolve the Knudsen layer, which is quite a bit wider than the interface.

It is well known that NSF and G13 models do not exhibit Knudsen layers, hence they are not suited to this task. Indeed, NSF jump and slip boundary conditions are often adjusted with Knudsen layer correction factors to account for this deficiency [13]. The same is not possible in the EV phase field model, where no correctable interface conditions exist. In classical kinetic theory, the first Grad-type moment model that has a full set of Knudsen layers is the Grad 26 moment model, which can be reduced to the regularized 13 moment equations which retains all Knudsen layer contributions [8]. Hence, our long term goal is the derivation of the corresponding 26 moment equations for the $\mathrm{EV}$ equation, and then further reducing these to the corresponding EV-R13 equations.

As an intermediate step towards this goal, we present here the EV-G13 equations for the Enskog-Vlasov equation, and first preliminary solutions of the equations in comparison to full solutions of the Enskog-Vlasov equation. Compared to the work by Kremer and Rosa [11], we not only add the Vlasov terms describing long range attractive forces-and hence phase change and interfaces-, but also consider higher orders in the Taylor expansion of the Enskog collision term. As Kremer and Rosa, we are interested in small deviations from equilibrium, which allows linearization of the equations around the equilibrium state. For this we have to consider an equilibrium state with vapor-liquid phase interface, where the equilibrium density gradient is quite steep. Hence, the linearized equations contain terms which are non-linear in the density gradient.

Due to space limitation, we do not present details of the procedures, but present only the resulting equations; a more complete account is planned for the future.

\section{ENSKOG-VLASOV EQUATION}

The Enskog-Vlasov equation is a modification of the Boltzmann equation to account for long-range forces between particles through the Vlasov force $F_{k}\left(x_{l}\right)$, and for finite particle volume through the Enskog collision term $\mathcal{S}_{\text {En }}(f, f)$. Following [3,4], we write the EV equation as

$$
\frac{\partial f}{\partial t}+c_{k} \frac{\partial f}{\partial x_{k}}+\left[F_{k}\left(x_{l}\right)+G_{k}\right] \frac{\partial f}{\partial c_{k}}=\mathcal{S}_{\mathrm{En}}(f, f)
$$

where $\mathbf{x}, t$, and $\mathbf{c}$ denote space, time and microscopic velocity, $f(\mathbf{x}, t, \mathbf{c})$ is the distribution function, and $\mathbf{G}$ denotes the external body force, e.g., gravity. Vector components are denoted as $x_{k}, x_{r}, c_{r}$ etc.

\section{Enskog collision term}

The Enskog collision term describes the change of the distribution function due to binary collisions of a dense gas of hard sphere molecules of finite diameter $a$, it reads [2][14]

$$
\mathcal{S}_{\mathrm{En}}=a^{2} \iint_{0}^{2 \pi} \int_{0}^{\pi / 2}\left\{\begin{array}{c}
Y\left[n\left(x_{r}+\frac{a k_{r}}{2}\right)\right] f\left(x_{s}+a k_{s}, c_{s}^{1 \prime}\right) f\left(x_{t}, c_{t}^{\prime}\right) \\
-Y\left[n\left(x_{r}-\frac{a k_{r}}{2}\right)\right] f\left(x_{s}-a k_{s}, c_{s}^{1}\right) f\left(x_{t}, c_{t}\right)
\end{array}\right\} g \cos \theta \sin \theta d \theta d \varepsilon d \mathbf{c}^{1} .
$$

Here, $k_{k}=\{\cos \varepsilon \sin \theta, \sin \varepsilon \sin \theta, \cos \theta\}_{k}$ is the collision (unit) vector, $n=\int f d \mathbf{c}$ is the particle number density. The pair correlation function at contact, $Y[n]$, is here approximated as a local function of the reduced density $\eta=\frac{\pi a^{3} n}{6}$, and computed from Carnahan-Starling [12] hard spheres equation of state as: $Y[n]=\frac{1}{2} \frac{2-\eta}{(1-\eta)^{3}}$. In particular, the Enskog terms accounts for the spatial variation of the distribution function on the scale of the particle diameter: the colliding particles have centers at $x_{s}$ and $x_{s} \pm a k_{s}$, with different values of the distribution at those points. The correlation function $Y[n]$ considers not only the eigenvolume of the particles, which reduces the accessible volume and thus increases the 
collision frequency, but also the reduced probability of collision due to screening (i.e., a closely neighboring particle blocks access to the collision partner for a colliding particle) [14]. In the limit of negligible reduced density, $\eta \rightarrow 0$, the Enskog term reduces to the classical Boltzmann collision term.

\section{Vlasov Force and Korteweg Stress}

The Vlasov force considers the mean force exerted on a particle at location $\mathbf{x}$ from particles at all other locations $\mathbf{x}^{1}$, based on the number density distribution $n\left(\mathbf{x}^{1}\right)$ and neglecting spatial correlations. With the particle interaction potential $\phi(r)$, the resulting force is

$$
F_{k}\left(x_{l}\right)=\int_{\left\|\mathbf{x}^{1}-\mathbf{x}\right\|>a} \frac{d \phi}{d r} \frac{x_{k}^{1}-x_{k}}{\left|\mathbf{x}^{1}-\mathbf{x}\right|} n\left(x_{r}^{1}\right) d \mathbf{x}^{1},
$$

where $r=\left|\mathbf{x}^{1}-\mathbf{x}\right|$ is the distance between two interacting particles. Since the Enskog term considers hard sphere particles, a good choice for the potential is

$$
\phi(r)=\left\{\begin{array}{cc}
\infty & r<a \\
-\phi_{a}\left(\frac{r}{a}\right)^{-\gamma} & r \geq a
\end{array}, \quad \phi_{a}>0\right.
$$

which describes hard sphere collisions when the particles meet at distance $a$, and an attractive power potential $\phi(r)$ when they are apart. It is to be noted that the algebraic form of the attractive potential tail in Eq. 4 results from a specific choice made here, not from a constraint of the theory. Since the attractive force decays fast with distance, and we expect smooth densities, we can employ the smooth density approximation, which results from Taylor expansion in $a$ as

$$
\begin{aligned}
F_{k}\left(x_{l}\right) & =\frac{\partial n}{\partial x_{s}} \int_{r>a} \frac{d \phi}{d r} \frac{r_{k} r_{s}}{r} d \mathbf{r}+\frac{1}{6} \frac{\partial n}{\partial x_{r} \partial x_{s} \partial x_{t}} \int_{r>a} \frac{d \phi}{d r} \frac{r_{k} r_{r} r_{s} r_{t}}{r} d \mathbf{r} \\
& =\frac{4 \pi a^{3}}{3} \frac{\gamma \phi_{a}}{\gamma-3}\left(\frac{\partial n}{\partial x_{k}}+\frac{a^{2}}{10} \frac{\gamma-3}{\gamma-5} \frac{\partial^{3} n}{\partial x_{k} \partial x_{s} \partial x_{s}}\right)
\end{aligned}
$$

The corresponding momentum supply $\rho F_{k}=m n F_{k}$ ( $\rho$ is mass density, and $m$ is the mass of a particle) can be expressed as the divergence of the Korteweg stress tensor $\mathcal{T}_{k s}^{K}$, that is

$$
\rho F_{k}=-\frac{\partial \mathcal{T}_{k s}^{K}}{\partial x_{s}}
$$

with

$$
\mathcal{T}_{k s}^{K}=-\frac{2 \pi}{3} \frac{a^{3}}{m} \frac{\gamma \phi_{a}}{\gamma-3} \rho^{2} \delta_{k s}-\frac{2 \pi}{15} \frac{a^{5}}{m} \frac{\gamma \phi_{a}}{\gamma-5}\left[\left(\rho \frac{\partial^{2} \rho}{\partial x_{r} \partial x_{r}}+\frac{1}{2} \frac{\partial \rho}{\partial x_{r}} \frac{\partial \rho}{\partial x_{r}}\right) \delta_{k s}-\frac{\partial \rho}{\partial x_{s}} \frac{\partial \rho}{\partial x_{k}}\right] .
$$

Here, the first term describes the pressure reduction due to attractive forces, just as it appears in the van der Waals equation [15]. The second term describes the capillary forces due to density gradients, including surface tension in the liquid-vapor interface, where the density gradient is steep [16].

The smooth density approximation poses strong restrictions on the density, as discussed in [17]. However, these restrictions do not affect the above approximation, which describes capillary stresses well [16].

\section{Goals}

Just as the Boltzmann equation, the Enskog-Vlasov equation is a high dimensional integro-differential equation, with an H-theorem. Typically, its solution relies on DSMC-like particle schemes, see, e.g., [18, 5].

Our goal is to approximate the EV equation by a low-dimensional set of macroscopic transport equations, just as in the moment method for the Boltzmann equation $[8,9,10]$. In particular, we aim to resolve the diffusive liquidvapor interface, and to analyze the macroscopic equations to gain insight into transport processes with evaporation and condensation. With the full resolution of the liquid-vapor interface, the equations will give insight into the elusive condensation coefficient [19], and, if a sufficient number of moments is considered, they will explicitly describe the interplay of diffusive interface and Knudsen layers. 


\section{MOMENT EQUATIONS}

\section{Grad's method of moments}

The aim of the moment method is to replace the detailed kinetic equation through a set of moment equations, that describe the main characteristics of the kinetic equation [10,8]. In general notation, we define a set of $N$ moments as

$$
u_{A}=\int \varphi_{A}\left(c_{i}\right) f d \mathbf{c}, \quad A=1, \ldots, N
$$

where the $\varphi_{A}\left(c_{i}\right)$ are suitable polynomials in the microscopic velocity that generate the moments. Multiplication of the kinetic equation (1) with $\varphi_{A}$ and integration over the microscopic velocity generates a set of moment equations

$$
\frac{\partial u_{A}}{\partial t}+\frac{\partial V_{A k}}{\partial x_{k}}=\left(F_{k}+G_{k}\right) U_{A k}+P_{A} \quad, A=1, \ldots, N
$$

where

$$
V_{A k}=\int \varphi_{A}\left(c_{i}\right) c_{k} f d \mathbf{c}, \quad U_{A k}=\int \frac{\partial \varphi_{A}\left(c_{i}\right)}{\partial c_{k}} f d \mathbf{c}, P_{A}=\int \varphi_{A}\left(c_{i}\right) \mathcal{S}_{\mathrm{En}}(f, f) d \mathbf{c} .
$$

While the $U_{A k}$ are directly related to lower order moments (see [8]), in order to obtain a closed set of equations, constitutive relations are required to link the fluxes $V_{A k}$ and the productions $P_{A}$ to the moments $u_{A}$, which are the variables of the system of equations.

Grad solved the closure problem by constructing a distribution function that depends explicitely on the moments [10], written as a disturbance of the equilibrium solution,

$$
f_{\mathrm{G}} \simeq f_{\mid E}\left[1-\lambda_{A}\left(u_{B}\right) \varphi_{A}\left(c_{i}\right)\right]
$$

Here, $f_{\mid E}=\frac{\rho}{m} \sqrt{\frac{m}{2 \pi k T}}^{3} \exp \left[-\frac{m C^{2}}{2 k_{B} T}\right]$ denotes the local Maxwellian equilibrium distribution, and the $\lambda_{A}$ are expansion coefficients (also, Boltzmann constant $k_{B}$, thermodynamic temperature $T$, peculiar velocity $C_{i}=c_{i}-v_{i}$, macroscopic velocity $v_{i}$ ). Insertion of the Grad distribution (11) into the moment definition (8) identifies the coefficients $\lambda_{A}$ as functions of the moments $u_{A}$, and then its use in the expressions (10) gives explicit constitutive relations of the form $V_{A k}\left(u_{B}\right), U_{A k}\left(u_{B}\right), P_{A}\left(u_{B}\right)$. Inserting these into (9) finally gives the explicit Grad moment system for the chosen set of moments. For further discussion on, e.g., the number of moments, alternative closures etc, we refer the reader to the scientific literature $[8,9,10]$.

While Grad performed the closure on moment equations for the Boltzmann equation, it can be also be applied to the EV equation, since the Maxwellian describes the local equilibrium state for the EV equation as well.

\section{Moment equations from Enskog-Vlasov}

We proceed with presenting the 13 moment equations obtained from the Enskog Vlasov equation by means of the Grad method. The 13 variables considered are those moments of the distribution function that have direct physical meaning,

$$
\begin{aligned}
& \rho=m n=m \int f d \mathbf{c}, \rho v_{i}=m \int c_{i} f d \mathbf{c}, \rho \epsilon=\frac{3}{2} \rho \theta=\frac{m}{2} \int C^{2} f d \mathbf{c} \\
& \sigma_{i j}=m \int C_{\langle i} C_{j\rangle} f d \mathbf{c}, \quad q_{i}=\frac{m}{2} \int C^{2} C_{i} f d \mathbf{c}
\end{aligned}
$$

Above, the first 5 moments, $\rho, \rho v_{i}$ and $\rho \epsilon$ are mass density, momentum density, and density of internal energy, which obey the appropriate conservation laws. $\theta=R T$ is the temperature in units of specific energy, where $R=k_{B} / m$ is the gas constant, $k_{B}$ is the Boltzmann constant, $m$ is particle mass, and $T$ is thermodynamic temperature. Stress tensor $\sigma_{i j}$ and heat flux $q_{i}$ are the kinetic contributions to overall stress and energy flux, there will be additional contributions from the Enskog and Vlasov terms that will emerge further below. Stress and heat flux are non-equilibrium moments, that is they vanish in equilibrium states; angular brackets indicate symmetric and trace free tensors [8]. 
While the computation of the fluxes $V_{A k}$ and the $U_{A k}$ is just as in the Boltzmann case, the computation of the productions $P_{A}$ from the Enskog collision term with the Grad distribution yields a complicated non-local expression,

$$
P_{A}=\int \varphi_{A}\left(c_{i}\right) a^{2} \iint_{0}^{2 \pi} \int_{0}^{\pi / 2}\left\{\begin{array}{c}
Y\left[n\left(x_{r}+\frac{a k_{r}}{2}\right)\right] f_{\mathrm{G}}\left(x_{s}+a k_{s}, c_{s}^{1 \prime}\right) f_{\mathrm{G}}\left(x_{t}, c_{t}^{\prime}\right) \\
-Y\left[n\left(x_{r}-\frac{a k_{r}}{2}\right)\right] f_{\mathrm{G}}\left(x_{s}-a k_{s}, c_{s}^{1}\right) f_{\mathrm{G}}\left(x_{t}, c_{t}\right)
\end{array}\right\} g \cos \theta \sin \theta d \theta d \varepsilon d \mathbf{c}^{1} d \mathbf{c}
$$

Note that the Grad distribution $f_{\mathrm{G}}$ depends on space and time only through the space-time dependence of the moments $u_{A}\left(x_{i}, t\right)$. Aiming for a macroscopic model in form of partial differential equations, we expand all non-localities into Taylor series, which gives gradient terms in all macroscopic quantities.

Specifically, we chose the particle diameter $a$ as the expansion parameter. Indeed, in the limit $a \rightarrow 0$ the Enskog collision term reduces to the Boltzmann collision term, hence the Taylor expansion in $a$ identifies the leading corrections to the classical Boltzmann theory.

For consistency with the Korteweg stress (7), where terms up to order $O\left(a^{5}\right)$ must be considered to describe surface tension in the phase interface, one might consider it necessary to expand the Enskog term to 5th order as well. However, in addition to the expansion in $a$, we are interested only in small deviations from an equilibrium state, where temperature and velocity are homogeneous, viscous stress and thermal heat flux vanish, while the density is in a non-homogeneous equilibrium state $\rho_{E}\left(x_{i}\right)$, which is affected by the 5 th order terms. We consider the deviation from equilibrium as another smallness parameter, and include only those 5th order terms in $a$ that do not vanish in equilibrium, while keeping 4th order terms that are multiplied with terms describing deviation from equilibrium (such as temperature gradients, stresses, etc). This distinguishes our work from that by Kremer \& Rosa [11], who did not consider the Vlasov forces, hence have no phase change, but only deal with the Enskog gas, so that they linearized in density gradients as well.

The Taylor expansion leads to a host of production terms, including contributions to conservation laws in divergence form, which are due to the transfer of momentum and energy in collisions between particles with centers at distance $a$.

\section{Closed 13 moment system for the Enskog-Vlasov equation}

Since no mass is transferred in collisions, the mass balance, or continuity equation, assumes the usual form [13]

$$
\frac{\partial \rho}{\partial t}+\frac{\partial \rho v_{k}}{\partial x_{k}}=0
$$

The balance of momentum is obtained as

$$
\frac{\partial \rho v_{i}}{\partial t}+\frac{\partial}{\partial x_{k}}\left(\begin{array}{c}
\rho v_{i} v_{k} \\
+\left[\rho \theta\left(1+\frac{2 \pi}{3} \frac{a^{3}}{m} \rho Y_{0}\right)-\frac{2 \pi a^{3}}{3} \frac{\gamma \phi_{a} / m}{\gamma-3} \rho^{2}\right] \delta_{i k} \\
+\left[1+\frac{2}{5}\left(\frac{2 \pi}{3} \frac{a^{3}}{m} \rho Y_{0}\right)\right] \sigma_{i k} \\
-\frac{4}{5} \frac{a^{4} \sqrt{\pi}}{m \sqrt{\theta}} \rho^{2} Y_{0}\left(\theta \frac{\partial \delta_{(i k} v_{s}}{\partial x_{s}}+\frac{1}{10} \frac{\partial_{\left(i k q_{s}\right)}}{\partial x_{s}}\right) \\
+\frac{\pi}{60} \frac{a^{5}}{m} \theta\left(\frac{\partial^{2} Y_{0} \rho^{2}}{\partial x_{s} x_{t}}+3 Y_{0} \frac{\partial^{2} \rho^{2}}{\partial x_{s} \partial x_{t}}-12 Y_{0} \frac{\partial \rho}{\partial x_{s}} \frac{\partial \rho}{\partial x_{t}}\right) \delta_{(i k} \delta_{s t)} \\
-\frac{2 \pi}{15} \frac{a^{5}}{m} \frac{\gamma \phi_{a}}{\gamma-5}\left[\left(\rho \frac{\partial^{2} \rho}{\partial x_{r} \partial x_{r}}+\frac{1}{2} \frac{\partial \rho}{\partial x_{r}} \frac{\partial \rho}{\partial x_{r}}\right) \delta_{i k}-\frac{\partial \rho}{\partial x_{i}} \frac{\partial \rho}{\partial x_{k}}\right]
\end{array}\right)=\rho G_{i}
$$

Indices in brackets indicate fully symmetric tensors. Here, the expression under the divergence is the overall momentum flux, which consists of a number of contributions. The first line is the usual convective transfer of momentum. The expression in the 2 nd line is the bulk pressure, which can be considered as a van der Waals like correction of the ideal gas pressure $p_{\mathrm{ig}}=\rho \theta$. The third and fourth lines describe viscous and thermal stresses at different orders in $a$. The last line is the capillary stress from the Kortweg stress tensor, while the second to last line is a correction stemming from the Enskog term. For an isothermal equilibrium case, the above equation reduces to the equations in [5].

The full energy balance accounts not only for thermal and kinetic energy, but has an additional contribution $\epsilon_{K}$ that accounts for the energy associated with the Vlasov force [16]. Written in balance law form, the conservation law 
for total energy reads

$$
\frac{\partial \rho\left(\frac{3}{2} \theta+\frac{1}{2} v^{2}+\epsilon_{K}\right)}{\partial t}+\frac{\partial}{\partial x_{k}}\left(\begin{array}{c}
{\left[\frac{\rho}{2} v^{2}+\rho \theta\left(\frac{5}{2}+\frac{2 \pi}{3} \frac{a^{3}}{m} \rho Y_{0}\right)\right] v_{k}} \\
+\left(1+\frac{3}{5} \frac{2 \pi}{3} \frac{a^{3}}{m} \rho Y_{0}\right) q_{k}+q_{k}^{K} \\
+\left[1+\frac{2}{5} \frac{2 \pi}{3} \frac{a^{3}}{m} \rho Y_{0}\right] \sigma_{i k} v_{i} \\
-\left[\left(2 \frac{2 \pi a^{3}}{3} \frac{\gamma \phi_{a} / m}{\gamma-3} \rho^{2} \delta_{i k}+\frac{2 \pi 5^{5}}{15} \frac{\gamma \phi_{a} / m}{\gamma-5}\left[\rho \frac{\partial^{2} \rho}{\partial x_{r} \partial x_{r}} \delta_{i k}-\frac{\partial \rho}{\partial x_{i}} \frac{\partial \rho}{\partial x_{k}}\right]\right) v_{i}\right]
\end{array}\right)=\rho v_{i} G_{i}
$$

The expression under the divergence is the overall energy flux, with convective and non-convective contributions, and the right hand side is the power associated with the external body forces. Here, we have introduced abbreviations for Korteweg energy and heat flux,

$$
\epsilon_{K}=-\frac{2 \pi a^{3}}{3} \frac{\gamma \phi_{a} / m}{\gamma-3} \rho+\frac{\pi a^{5}}{15} \frac{\gamma \phi_{a} / m}{\gamma-5} \frac{1}{\rho} \frac{\partial \rho}{\partial x_{s}} \frac{\partial \rho}{\partial x_{s}} \quad, \quad q_{i}^{K}=\frac{2 \pi a^{5}}{15} \frac{\gamma \phi_{a} / m}{\gamma-5} \rho \frac{\partial \rho}{\partial x_{i}} \frac{\partial v_{r}}{\partial x_{r}}
$$

The first term in $\epsilon_{K}$ is well known as the energy correction for the van der Waals gas [15].

By combining the energy balance with the conservation laws for mass and momentum, we find the balance of thermal energy as

$$
\begin{array}{r}
\frac{3}{2} \rho \frac{D \theta}{D t}+\frac{\partial}{\partial x_{k}}\left[\left(1+\frac{3}{5} \frac{2 \pi}{3} \frac{a^{3}}{m} \rho Y_{0}\right) q_{k}-\frac{2}{3} \frac{a^{4} \sqrt{\pi}}{m \sqrt{\theta}} \rho^{2} Y_{0} \theta\left(\frac{\partial \theta}{\partial x_{k}}+\frac{2}{5} \frac{\partial}{\partial x_{s}}\left(\frac{\sigma_{k s}}{\rho}\right)\right)\right] \\
=-\left[\rho \theta\left(1+\frac{2 \pi}{3} \frac{a^{3}}{m} \rho Y_{0}\right) \delta_{k l}+\left(1+\frac{2}{5} \frac{2 \pi}{3} \frac{a^{3}}{m} \rho Y_{0}\right) \sigma_{k l}\right] \frac{\partial v_{k}}{\partial x_{l}}
\end{array}
$$

Here, the expression under the divergence is the non-convective thermal heat flux $Q_{k}$, and the right hand side is the frictional heating.

The conservation laws for mass, momentum and energy contain the viscous stress $\sigma_{i j}$ and the heat flux $q_{i}$, for which the Grad method with 13 moments gives the closed balance equations

$$
\begin{aligned}
& \frac{D \sigma_{i j}}{D t}+2\left[\rho \theta\left(1+\frac{2}{5} \frac{2 \pi}{3} \frac{a^{3}}{m} \rho Y_{0}\right)\right] \frac{\partial v_{\langle i}}{\partial x_{j\rangle}}+\frac{4}{5} \frac{\partial}{\partial x_{\langle i}}\left[\left(1+\frac{2 \pi}{3} \frac{3}{5} \frac{a^{3}}{m} \rho Y_{0}\right) q_{j\rangle}\right] \\
& +\frac{6}{25} \frac{2 \pi}{3} \frac{a^{3}}{m}\left[q_{\langle i} \delta_{j\rangle r}\right] \frac{1}{\rho} \frac{\partial \rho^{2} Y_{0}}{\partial x_{r}}-\frac{8}{5} \frac{a^{4} \sqrt{\pi}}{m \sqrt{\theta}} \frac{\partial}{\partial x_{\langle i}}\left[\rho^{2} Y_{0} \frac{\partial \theta}{\partial x_{j\rangle}}\right] \\
& -\frac{4}{105} \frac{a^{4} \sqrt{\pi}}{m \sqrt{\theta}}\left\{\begin{array}{r}
2 \rho \theta \frac{\partial}{\partial x_{r}}\left(Y_{0} \frac{\partial \sigma_{i j}}{\partial x_{r}}\right)-12 \theta \sigma_{i j} \frac{\partial}{\partial x_{r}}\left(Y_{0} \frac{\partial \rho}{\partial x_{r}}\right)-\frac{5}{2} \rho \theta \sigma_{i j} \frac{\partial^{2} Y_{0}}{\partial x_{r} \partial x_{r}} \\
+8 \rho \theta \frac{\partial}{\partial x_{r}}\left(Y_{0} \frac{\partial \sigma_{r i}}{\partial x_{j\rangle}}\right)-20 \theta \sigma_{r\langle i} \frac{\partial}{\partial x_{r}}\left(Y_{0} \frac{\partial \rho}{\partial x_{j\rangle}}\right)-3 \rho \theta \sigma_{r\langle i} \frac{\partial^{2} Y_{0}}{\partial x_{j} \partial x_{r}}
\end{array}\right\}=-\frac{16}{5} \frac{\rho \sqrt{\pi \theta} a^{2}}{m} Y_{0} \sigma_{i j} \\
& \frac{D q_{i}}{D t}+\frac{5}{2} \rho \theta\left[1+\frac{3}{5} \frac{2 \pi}{3} \frac{a^{3}}{m} \rho Y_{0}\right] \frac{\partial \theta}{\partial x_{i}}+\theta \frac{\partial}{\partial x_{k}}\left[\left(1+\frac{3}{5} \frac{2 \pi}{3} \frac{a^{3}}{m} \rho Y_{0}\right) \sigma_{i k}\right] \\
& -\frac{\sigma_{i k} \theta}{\rho} \frac{\partial}{\partial x_{k}}\left[\rho\left(1+\frac{9}{10} \frac{2 \pi}{3} \frac{a^{3}}{m} \rho Y_{0}\right)\right]-\frac{a^{4} \rho \sqrt{\pi \theta}}{m} \frac{\theta}{\rho}\left\{\frac{407}{90} \frac{\partial}{\partial x_{r}}\left(\rho^{2} Y_{0} \frac{\partial v_{i}}{\partial x_{r}}\right)+\frac{87}{15} \frac{\partial}{\partial x_{(r}}\left(\rho^{2} Y_{0} \frac{\partial v_{r}}{\left.\partial x_{i}\right)}\right)\right\} \\
& -\frac{a^{4} \rho \sqrt{\pi \theta}}{m} \frac{1}{5}\left\{\begin{array}{c}
{\left[\frac{151}{18} \frac{\partial}{\partial x_{r}}\left(Y_{0} \frac{\partial q_{i}}{\partial x_{r}}\right)-\frac{373}{45} \frac{q_{i}}{\rho} \frac{\partial}{\partial x_{r}}\left(Y_{0} \frac{\partial \rho}{\partial x_{r}}\right)-\frac{7}{30} \frac{\partial^{2} Y_{0}}{\partial x_{r} \partial x_{r}} q_{i}\right]} \\
+\left[18 \frac{\partial}{\partial x_{(r}}\left(Y_{0} \frac{\partial q_{r}}{\left.\partial x_{i}\right)}\right)-\frac{407}{15} \frac{q_{r}}{\rho} \frac{\partial}{\partial x_{(r}}\left(Y_{0} \frac{\partial \rho}{\partial x_{i)}}\right)-\frac{2}{15} \frac{\partial^{2} Y_{0}}{\partial x_{r} \partial x_{i}} q_{r}\right]
\end{array}\right\}=-\frac{2}{3} \frac{16}{5} \frac{\rho \sqrt{\pi \theta} a^{2}}{m} Y_{0} q_{i}
\end{aligned}
$$

We emphasize, that $\sigma_{i j}=m \int C_{\langle i} C_{j\rangle} f d \mathbf{c}$ and $q_{i}=\frac{m}{2} \int C^{2} C_{i} f d \mathbf{c}$ are not the full stress tensor and heat flux, which we have identified in the conservation laws for momentum and energy above. 


\section{Navier-Stokes and Fourier laws}

Performing a Chapman-Enskog expansion [8] on the balances for $\sigma_{i j}$ and $q_{i}$ for bulk phases, we find their leading order contributions as

$$
\sigma_{i k}=-2 \frac{\left[\rho \theta\left(1+\frac{2}{5} \frac{2 \pi}{3} \frac{a^{3}}{m} \rho Y_{0}\right)\right]}{\frac{16}{5} \frac{\rho}{m} \sqrt{\pi \theta} a^{2} Y_{0}} \frac{\partial v_{\langle i}}{\partial x_{k\rangle}}, q_{i}=-\frac{15}{4} \frac{\rho \theta\left[1+\frac{3}{5} \frac{2 \pi}{3} \frac{a^{3}}{m} \rho Y_{0}\right]}{\frac{16}{5} \frac{\rho}{m} \sqrt{\pi \theta} a^{2} Y_{0}} \frac{\partial \theta}{\partial x_{i}}
$$

In order to have the complete viscous stresses and thermal heat flux, we have to insert the above expressions into the fluxes of momentum and energy as identified in the energy and momentum balances $(15,18)$. Looking only at the terms that describe viscosity effects, we identify the viscous stresses in the Navier-Stokes form

$$
\Pi_{i k}=-2 \mu \frac{\partial v_{\langle i}}{\partial x_{k\rangle}}-v \frac{\partial v_{s}}{\partial x_{s}} \delta_{i k}
$$

where the shear viscosity $\mu$ and the bulk viscosity $v$ are given by

$$
\mu=\rho \theta \frac{\left(1+\frac{2}{5} \frac{2 \pi}{3} \frac{a^{3}}{m} \rho Y_{0}\right)^{2}}{\frac{16}{5} \frac{\rho}{m} \sqrt{\pi \theta} a^{2} Y_{0}}+\frac{4}{15} \frac{a^{4} \sqrt{\pi \theta}}{m} \rho^{2} Y_{0} \quad, \quad v=\frac{4}{9} \frac{a^{4} \sqrt{\pi \theta}}{m} \rho^{2} Y_{0}
$$

Similarly, inserting the approximation for $q_{i}$ in the thermal energy flux, we find the Fourier law

$$
Q_{k}=-\kappa \frac{\partial \theta}{\partial x_{k}}
$$

with the heat conductivity

$$
\kappa=\frac{15}{4} \frac{\rho \theta\left(1+\frac{3}{5} \frac{2 \pi}{3} \frac{a^{3}}{m} \rho Y_{0}\right)^{2}}{\frac{16}{5} \frac{\rho}{m} \sqrt{\pi \theta} a^{2} Y_{0}}+\frac{2}{3} \frac{a^{4} \sqrt{\pi \theta}}{m} \rho^{2} Y_{0}
$$

We note that these expressions for the transport coefficients agree with [14], where they were obtained by CE expansion of the Enskog-Vlasov equation itself, while here they follow from CE expansion of the moment equations.

\section{Application: Two-phase Couette flow}

In the model problem described in this section, the fluid, described by Eqs. (14-20), occupies the half-space $x_{1}>0$. The fluid region consists of a liquid film of finite thickness and is located in the strip $0<x_{1}<x_{L}$, the liquid vapor interface, of nominal thickness $\Delta_{I}\left(\Delta_{I} \ll x_{L}\right)$, and the vapor region $x_{1}>x_{L}+\Delta_{I}$. A steady shear velocity $v_{2}\left(x_{1}\right)$, parallel to the interface, is imposed to the fluid. It is assumed that $v_{2}(0)=0$ and $\lim _{x_{1} \rightarrow \infty} \frac{d v_{2}}{d x_{1}}=s_{21}$, with $s_{21}$ constant. In the limit of small shear rate $s_{21}$, it is possible to linearize the transport equations around the equilibrium state at temperature $T<T_{c}$. It is also possible to show that, to first order in $s_{21}$, one has $T\left(x_{1}\right)=T$ and the density profile coincides with the equilibrium one. The latter can be obtained by the $x_{1}$ component of Eq. (15) whose equilibrium form can be easily recast as follows:

$$
\begin{gathered}
p(\rho, \theta)+\alpha(\rho, \theta)\left(\frac{d \rho}{d x_{1}}\right)^{2}+\beta(\rho, \theta)\left(\frac{d^{2} \rho}{d x_{1}^{2}}\right)=p_{0} \\
\alpha(n, T)=\frac{\pi a^{5}}{15}\left[k_{B} T\left(n \frac{d Y}{d n}+\frac{n^{2}}{4} \frac{d^{2} Y}{d n^{2}}-Y\right)+\frac{\gamma}{\gamma-5} \phi_{a}\right], \quad \beta(n, T)=\frac{2 \pi a^{5}}{15} n\left[k_{B} T\left(Y+\frac{n}{8} \frac{d Y}{d n}\right)-\frac{\gamma}{\gamma-5} \phi_{a}\right]
\end{gathered}
$$

where $p(\rho, \theta)$ is the pressure in the uniform equilibrium state, whereas $\alpha(\rho, \theta)$ and $\beta(\rho, \theta)$ are related to capillary forces showing up when density is not constant. Their expressions are derived in Ref. [5]. Once $T$ is assigned, the equilibrium density profile $\rho_{0}\left(x_{1}\right)$, as well as the equilibrium pressure $p_{0}$ can be determined by solving the resulting differential equation [5]. In order to have a more manageable expression for $\rho_{0}\left(x_{1}\right)$, the latter has been approximated by a tanh profile. Figures $1 \mathrm{a}$ and $1 \mathrm{~b}$, compare the tanh approximation of equilibrium density profiles with the corresponding 

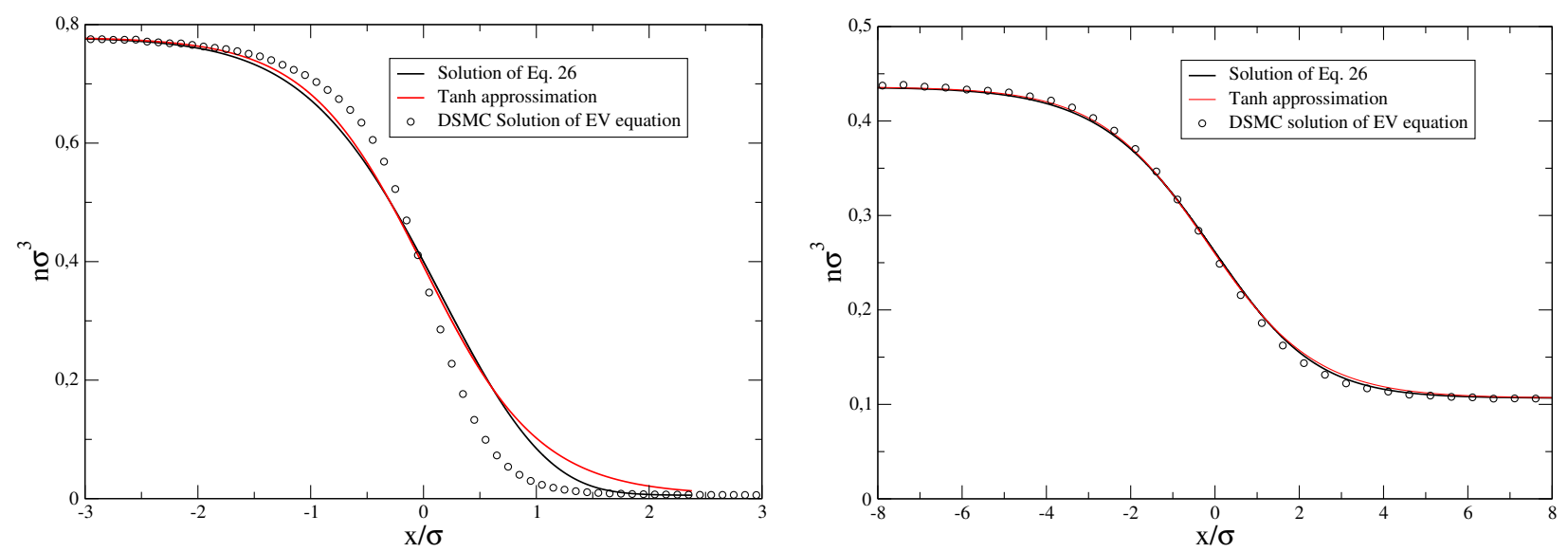

FIGURE 1. Left- Equilibrium reduced density profiles $k_{B} T_{0} / \phi_{a}=0.45$ Right- Equilibrium reduced density profiles $k_{B} T_{0} / \phi_{a}=$ 0.70

profile obtained by solving Eq. 26. The density profiles show that the tanh approximation performes well and becomes better when $T$ approaches the critical temperature, $T_{c}$. Further comparisons with DSMC solutions of $E V$ equations also show that, as noticed in Ref. [5], the actual $E V$ density profiles are generally steeper than those provided by Korteweg local approximation of non-local capillary stresses.

Since we impose constant temperature, the energy balance is not relevant, and the flow field $v_{2}(x)$ is determined through the equations for conservation of total tangential momentum $P_{12}$

$$
\left(1+\frac{2}{5} \frac{2 \pi}{3} \frac{a^{3}}{m} \rho Y_{0}\right) \sigma_{12}-\frac{4}{15} \frac{a^{4} \rho \sqrt{\pi \theta}}{m} Y_{0}\left(\rho \frac{\partial v_{2}}{\partial x}+\frac{1}{10} \frac{\rho}{\theta} \frac{\partial}{\partial x}\left(\frac{q_{2}}{\rho}\right)\right)=P_{12}
$$

shear stress balance

$$
\begin{aligned}
& {\left[\rho \theta\left(1+\frac{2}{5} \frac{2 \pi}{3} \frac{a^{3}}{m} \rho Y_{0}\right)\right] \frac{\partial v_{2}}{\partial x}+\frac{2}{5} \frac{\partial}{\partial x}\left[\left(1+\frac{2 \pi}{3} \frac{3}{5} \frac{a^{3}}{m} \rho Y_{0}\right) q_{2}\right]+\frac{6}{25} \frac{2 \pi}{3} \frac{a^{3}}{m}\left[\frac{1}{2} q_{2}\right] \frac{1}{\rho} \frac{\partial \rho^{2} Y_{0}}{\partial x}} \\
& -\frac{4}{105} \frac{\rho a^{4} \sqrt{\theta \pi}}{m}\left\{6 \frac{\partial}{\partial x}\left(Y_{0} \frac{\partial \sigma_{12}}{\partial x}\right)-22 \frac{\sigma_{12}}{\rho} \frac{\partial}{\partial x}\left(Y_{0} \frac{\partial \rho}{\partial x}\right)-4 \sigma_{12} \frac{\partial^{2} Y_{0}}{\partial x \partial x}\right\}=-\frac{16}{5} \frac{\rho \sqrt{\pi \theta} a^{2}}{m} Y_{0} \sigma_{12}
\end{aligned}
$$

and heat flux balance

$$
\begin{aligned}
& \theta \frac{\partial}{\partial x}\left[\left(1+\frac{3}{5} \frac{2 \pi}{3} \frac{a^{3}}{m} \rho Y_{0}\right) \sigma_{12}\right]-\frac{a^{4} \rho \sqrt{\pi \theta}}{m} \frac{\theta}{\rho} \frac{407}{90} \frac{\partial}{\partial x}\left(\rho^{2} Y_{0} \frac{\partial v_{2}}{\partial x}\right)-\frac{a^{4} \rho \sqrt{\pi \theta}}{m} \frac{1}{5}\left\{\frac{151}{18} \frac{\partial}{\partial x}\left(Y_{0} \frac{\partial q_{2}}{\partial x}\right)\right. \\
& \left.-\frac{373}{45} \frac{q_{2}}{\rho} \frac{\partial}{\partial x}\left(Y_{0} \frac{\partial \rho}{\partial x}\right)-\frac{7}{30} \frac{\partial^{2} Y_{0}}{\partial x \partial x} q_{2}\right\}-\frac{\sigma_{12} \theta}{\rho} \frac{\partial}{\partial x}\left[\rho+\frac{9}{10} \frac{2 \pi}{3} \frac{a^{3}}{m} \rho^{2} Y_{0}\right]=-\frac{2}{3} \frac{16}{5} \frac{\rho \sqrt{\pi \theta} a^{2}}{m} Y_{0} q_{2}
\end{aligned}
$$

If the constant shear stress $P_{12}$ is assigned, boundary conditions, at $x_{1}=0$ and $x_{1}=L$ are specified as follows: the liquid is at rest at the left boundary, $v_{2}(0)=0$; in the vapor bulk, up to the right boundary at $x_{1}=L$, one obtains the velocity gradient from $P_{12}=-\mu(\rho, T) \frac{\partial v_{2}}{\partial x_{1}}$, being $\mu(\rho, T)$ the viscosity coefficient resulting from Eqs. (28,29). The same equations provide the constant values the kinetic shear stress $\sigma_{12}$ takes in the uniform density regions to be used as boundary values. Since both domain boundaries belong to the hydrodynamic flow region, one has $q_{2}(0)=q_{2}(L)=0$, for the parallel heat flux.

Equation (28-30) constitute a system of linear first and second order differential equation which has been solved numerically by an iterative method, based on a simple finite difference discretization. Preliminary results have been compared with DSMC solution of the full Enskog-Vlasov equation. Figure 2 reports such comparison for a case in which $\phi_{a} / \theta=0.5$ and $\gamma=6$; the selected temperature corresponding to $2 / 3$ of the critical value. Liquid and vapor bulk densities in equilibrium are $\rho_{L}=0.7187 \frac{\mathrm{m}}{a^{3}}, \rho_{V}=0.012313 \frac{\mathrm{m}}{a^{3}}$, respectively. The comparison shows that including $q_{2}$ 


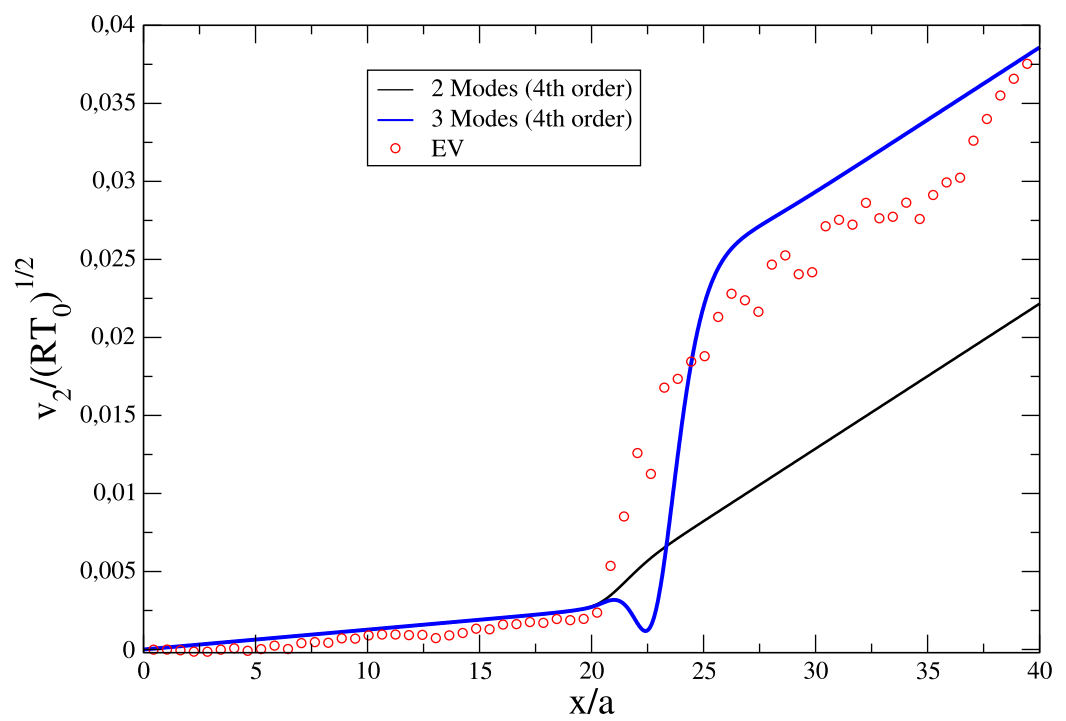

FIGURE 2. Velocity profiles for isothermal Couette flow parallel to a phase interface centered at $x / a \simeq 20$. Results shown are from a numerical solution of the Enskog-Vlasov equation (red dots), a reduced 2 mode model $\left(v_{2}, \sigma_{12}\right.$, black line) and the isothermal 13 moment 3 mode model $\left(v_{2}, \sigma_{12}, q_{2}\right.$, blue line).

into the picture provides a considerable improvement over the simpler approximation which takes into account only $v_{2}$ and $\sigma_{12}$. However, the agreement with the reference DSMC solution is still not satisfactory. The small unphysical velocity wiggle, in particular, might be caused by the approximations about the equilibrium density profile or might be a signal of the need to include additional moments.

\section{SUMMARY AND OUTLOOK}

In summary, we have embarked in developping, evaluating and solving moment equations for the Enskog-Vlasov equation. These moment equations not only describe liquid and vapor bulk phases, but fully resolve the liquid-vapor interface. Above, we have presented a closed set of 13 moment equations, with short discussions of equilibria, hydrodynamic transport coefficients and isothermal Couette flow. For the future, we plan further evaluation of equations with 13 and more moments, in particular by studying processes involving evaporation and condensation. Interesting theoretical questions to consider are, e.g., systematic limits to identify appropriate bulk and interface equations, the relation to jump conditions at the phase boundary, and the detailed comparison to direct solutions of the Enskog-Vlasov equation, in simple and complex geometries.

\section{REFERENCES}

[1] A.A. Vlasov, Many-Particle Theory and Its Application to Plasma, Gordon and Breach, 1961

[2] D. Enskog, The numerical calculation of phenomena in fairly dense gases, Arkiv Mat. Astr. Fys. 16(1) (1921)

[3] L. de Sobrino, On the Kinetic Theory of a van der Waals Gas, Can. J. Phys. 45, 363-385 (1967)

[4] M. Grmela, Kinetic Equation Approach to Phase Transitions, J. Stat. Phys. 3, 347-364 (1971)

[5] A. Frezzotti, L. Gibelli, and S. Lorenzani, Mean field kinetic theory description of evaporation of a fluid into vacuum, Phys. Fluids 17, 012102 (2005)

[6] E.S. Benilov, and M. S. Benilov, Energy conservation and H theorem for the Enskog-Vlasov equation, Phys. Rev E 97, 062115 (2018)

[7] S. Chapman and T. G. Cowling, The Mathematical Theory of Non-Uniform Gases. Cambridge University Press 1970

[8] H. Struchtrup, Macroscopic Transport Equations for Rarefied Gas Flows-Approximation Methods in Kinetic Theory. Interaction of Mechanics and Mathematics Series, Springer, Heidelberg 2005 
[9] M. Torrilhon, Modeling Nonequilibrium Gas Flow Based on Moment Equations. Annu. Rev. Fluid Mech. 48, 429-458 (2016)

[10] H. Grad, Principles of the Kinetic Theory of Gases, in Handbuch der Physik XII: Thermodynamik der Gase, S. Flügge (Ed.), Springer, Berlin 1958

[11] G.M. Kremer and E. Rosa Jr., On Enskog's dense gas theory. I. The method of moments for monatomic gases, J. Chem. Phys. 89, 3240-3247 (1988)

[12] N. F. Carnahan and K. E. Starling, Equation of State for Nonattracting Rigid Spheres, Journal of Chemical Physics 51, 635-636 (1969)

[13] C. Cercignani, Theory and Application of the Boltzmann Equation. Scottish Academic Press, Edinburgh 1975

[14] P. Resibois and M. De Leener, Classical Kinetic Theory of Fluids, Wiley, 1977

[15] H. Struchtrup, Thermodynamics and Energy Conversion (Springer, Heidelberg, 2014)

[16] D.M. Anderson, G.B. McFadden, and A.A. Wheeler, Diffusive-Interface Methods in Fluid Mechanics, Annu. Rev. Fluid Mech. 30, 139-165 (1998)

[17] M. Sadra and M.H. Gorji, Treatment of long-range interactions arising in the Enskog-Vlasov description of dense fluids, J. Comp. Phys. 378, 129-142 (2019)

[18] A. Frezzotti, A particle scheme for the numerical solution of the Enskog equation, Phys. Fluids 9, 1329-1335 (1997)

[19] R. Marek and J. Straub, Analysis of the evaporation coefficient and the condensation coefficient of water, Int. J. Heat and Mass Transfer, 44, 39-53 (2001) 